\title{
Echocardiographic and Angiographic Profile of Patients with Lateral Wall Mi Ecg Presentation in Inferior Wall Mi (A Comparison Study)
}

\author{
Sibaram Panda ${ }^{1 *}$, Sunil Kumar Sharma ${ }^{2}$
}

${ }^{1}$ Asst. Professors, ${ }^{2}$ Professor \& HOD, Dept. of Cardiology, VIMSAR, Burla, India

DOI: $10.36347 /$ sjams.2020.v08i11.029

| Received: 06.11.2020 | Accepted: 19.11.2020 | Published: 23.11.2020

*Corresponding author: Dr. Sibaram Panda

Abstract

Original Research Article

Background: Patient with lateral wall changes in inferior wall MI usually ignored most often. Patients with inferolateral MI show very diverse clinical presentations as compared to inferior wall MI without lateral MI ECG changes of inferolateral MI in comparison to inferior wall MI without lateral wall ECG changes. Materials and methods: This study enrolled 405 patients admitted to cardiology emergency with inferior wall MI. Patients divided into three groups on the basis of ECG changes, A) inferolateral B) isolated inferior wall MI MI C) inferior wall MI with RVMI. Patients with RVMI (group C) excluded from the group. Electrocardiographic, echocardiographic, angiographic outcomes compared between group A and B. Results: More than $1 / 3$ rd patient with inferior wall MI have associated lateral wall changes. STEMI equivalent changes in anterior leads noted in around $2 / 3 \mathrm{rd}$ patients. LV dysfunction seen in around $47.5 \%$ of the patient of inferolateral wall MI, which is significantly higher in comparison to $9 \%$ patients in inferior wall MI .Culprit lesion in inferolateral MI most often found to be left circumflex coronary artery. Multivessel lesion in inferolateral MI in 36.8\% patients, which is significantly higher as compared to inferior wall MI without lateral wall ECG changes (i.e.11.4\% patient). Conclusion: Patient with infererolateral MI have grave prognosis due to higher incidence of LV dysfunction, multivessel involvement and mitral regurgitation.Culprit lesion is most often left circumflex artery. So meticulous attention should be given to patients with lateral wall MI changes in inferior wall MI for better treatment strategy.

Keywords: Lateral wall MI, LV systolic function, regional wall motion abnormality, left circumflex Artery, Multivessel disease.

Copyright $\odot 2020$ The Author(s): This is an open-access article distributed under the terms of the Creative Commons Attribution 4.0 International License (CC BY-NC 4.0) which permits unrestricted use, distribution, and reproduction in any medium for non-commercial use provided the original author and source are credited.

\section{INTRODUCTION}

It is commonly accepted that the heart is located in the thorax strictly in a posteroanterior position; However present day CMR imaging reveals that heart sits on diaphragm in a slightly oblique rightto-left line. So it is postulated that RS pattern in V1 is accounted for by lateral and not inferobasal MI (classically posterior MI). At present newly revised AHA definition of lateral wall MI includesprominent $\mathrm{R}$ wave in leads in V1, V2 along with ST elevation in lead $\mathrm{I}$, avL and /or V5,V6. As a result horizon of lateral wall expanded with new definition [1, 2]. However lateral wall MI ECG changes all most commonly seen and most often ignored, when it has very dynamic impact on outcome of infererior wall MI. So study of echocardiography and angiographic profile very essential in the above group.

\section{Materials AND Methods}

Type of study - Prospective longitudinal case control study.

Place of study - VIMSAR, Burla.

Period of study $-15^{\text {th }}$ December 2019 to $15^{\text {th }}$ April 2020.

Inclusion criteria - interior wall STEMI patient below 18 yrs.

Total 427 patients with Inferior wall STEMI below 18years in admitted to VIMSAR, Burla enrolled in the study. Out of 427 patients 22 patients having associated anterior wall AMI (old), congenital heart disease, valvular heart disease, malignancy, chronic kidney disease, chronic lung disease, chronic liver disease, severe anaemia and patients with age more than 80 years are excluded. Consent taken from the patients enrolled in the study.

Out of 405 patients, 137 patients have ST elevation (I,avl),(V5,V6),(V7,V8,V9) or Q wave 
equivalent with ST depression in V1,V2 (i.e lateral wall MI)included in group A. 157 patients have ST elevation V3R,V4R (RVMI) associated with inferior wall MI included in group C.111 patients have inferior wall MI without lateral wall MI or RVMI ECG changes included in group B. Patients RVMI (group C) were excluded from the study.

ECG done 6hrly in every patient or as per the requirement needed. All patients routinely undergone echocardiography within $24 \mathrm{hr}$ of admission and parameters like EF, RWMA are taken into account.Patient with inferior wall $\mathrm{MI}$ are divided into groups(EF >50\%-normal, EF 40-50\%-mild, EF30-39\% moderate, EF $<30 \%$ severe ). Severity of LV dysfunction between two groups compared statistically. Incidence and pattern of RWMA compared between two groups. Number of lesions, culprit vessel noted in Patient undergoing angiography. To avoid confusion while finding the culprit vessel, single vessel disease lesion taken into account for the study. Incidence of multivessel disease compared statistically between two groups.

\section{Statistical ANALYSIS}

$\mathrm{P}$ value derived by comparing proportion of patients of two group statistically. $\mathrm{P}$ value $<0.05$ is significant.

Ethical approval - Ethical clearance taken from ethical committee

\section{RESULTS}

1)Out of 405 patients, it was seen 111 patients $(27.4 \%)$ have isolated inferior wall MI,137 patients $(33.8 \%)$ have lateral wall MI associated with inferior wall MI, 157 patients $(38.7 \%)$ have associated RVMI (Fig.1).

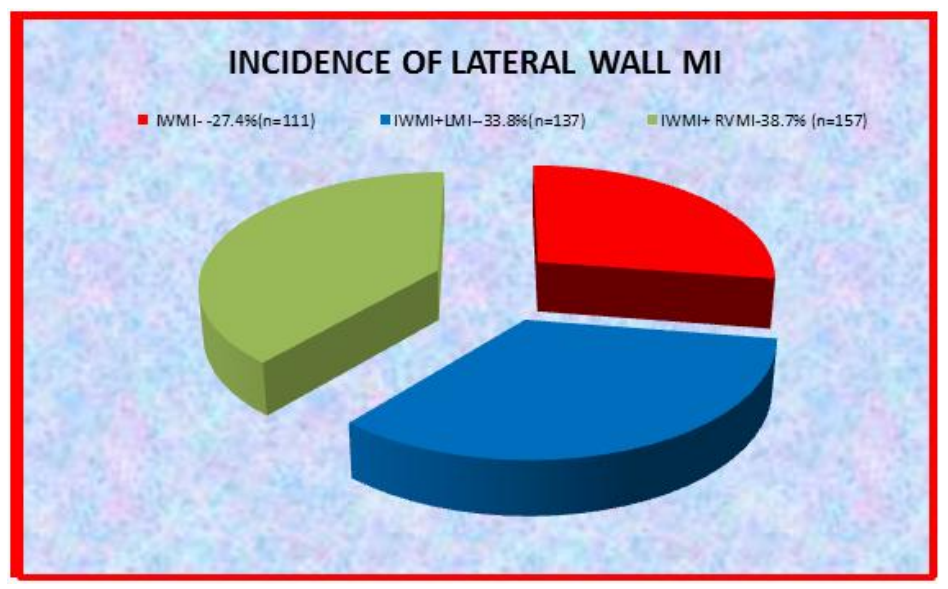

Fig-1: Incidence of lateral ami

2)In patients with lateral wall $\mathrm{MI}(137$ patients),ST elevation in V5,V6 or I.avl seen in 47 patients (35\%),ST depression with Qwave equivalent seen in 63 patients $(45.9 \%)$ and E.C.G changes in both group of leads found in only 26 (18.9\%)patients (Fig.2).

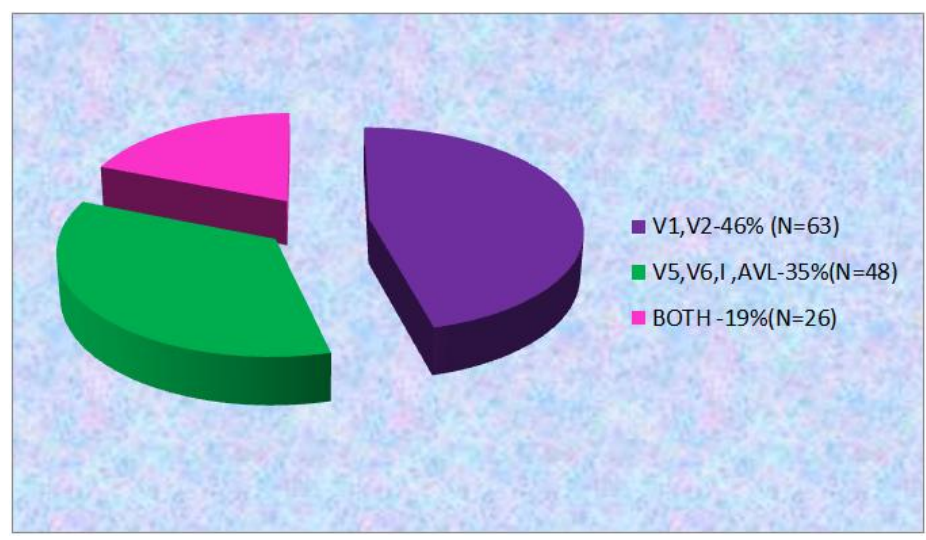

Fig-2: Distribution of lateral wall mi among ecg leads (n=137)

3) Around 66(45.9\%) patients in group-A have mild to moderate LV systolic dysfunction, where as in group B around $10(9 \%)$ patients have mild to moderate LV dysfunction. (Table 1) Mean EF in group A is
$48 \pm 8.9$ as compared group B $52 \pm 10.2$ with p value 0.0011 .Incidence of mitral regurgitation is significantly high in inferolateral group (Table 2). 
Table-1: Comparison of systolic function in inferolateral mi and inferior wall mi

\begin{tabular}{|l|c|c|c|c|c|}
\hline \multirow{2}{*}{ EF (\%) } & \multicolumn{2}{|c|}{ IWMI+LMI } & \multicolumn{2}{|c|}{ IWMI } & \multirow{2}{*}{ P VALUE } \\
\cline { 2 - 5 } & $(\mathbf{n o s})$ & $\boldsymbol{( \% )}$ & $\boldsymbol{( n o s )}$ & $(\boldsymbol{\%})$ & \\
\hline$>50$ & 71 & 51.8 & 101 & 90.9 & $<.0001$ \\
\hline $40-49$ & 41 & 29.9 & 9 & 8.1 & $<.0001$ \\
\hline $30-39$ & 22 & 16.0 & 1 & 0.9 & $<0.001$ \\
\hline$<30 \%$ & 3 & 2.1 & 0 & 0 & CANT BE COMPARED \\
\hline
\end{tabular}

Table-2: Comparison of mechanical complication in inferolateral mi and inferior wall mi

\begin{tabular}{|l|c|c|c|c|c|}
\hline \multirow{2}{*}{} & \multicolumn{2}{|c|}{ INFEROLATERAL MI } & \multicolumn{2}{c|}{ INFERIOR WALL MI } & \multirow{2}{*}{ P VALUE } \\
\cline { 2 - 6 } & No. & $\%$ & No. & $\%$ & \\
\hline MITRAL REGURGITATTION & 8 & 5.8 & 1 & 0.9 & 0.0396 \\
\hline VENTRICULAR SEPTAL RUPTURE & 1 & 0.72 & 0 & 0 & NS \\
\hline PERICARDIAL EFFUSION & 13 & 9.4 & 4 & 3.6 & NS \\
\hline LV ANEURYSM & 0 & 0 & 0 & 0 & NS \\
\hline
\end{tabular}

5) $70.8 \%$ patients with group A have shown RWMA in LCX territory as compared to group B
$(16.2 \%)$ patient in group B with $\mathrm{p}$ value $<0.001$. (Table 3)

Table-3: Relation between rwma $\&$ association of lateral wall mi

\begin{tabular}{|c|c|c|c|c|c|c|}
\hline \multirow[t]{2}{*}{ RWMA } & \multicolumn{2}{|c|}{ RCA TERRITORY } & \multicolumn{2}{|c|}{$\begin{array}{c}\text { LCX } \\
\text { TERRITORY }\end{array}$} & \multicolumn{2}{|c|}{ NO RWMA } \\
\hline & nos & $\%$ & nos & $\%$ & nos & $\%$ \\
\hline IWMI+LMI & 36 & 26.2 & 97 & 70.8 & 4 & 2.9 \\
\hline IWMI & 85 & 76.5 & 18 & 16.2 & 8 & 7.3 \\
\hline P VALUE & \multicolumn{2}{|c|}{$<0.001$} & \multicolumn{2}{|c|}{$<0.001$} & \multicolumn{2}{|c|}{$0.1094(\mathrm{NS})$} \\
\hline
\end{tabular}

6) Angiography done in 109 patients out of 137 patients in group A, whereas 96 patients out of 111 patients in group B undergone angiography. It is observed that SVD found in $63.2 \%(\mathrm{n}=69)$ patients in group A as compared $88.6 \%(n=85)$ patients in group $B$.
However most astonishing observation is that, multiple vessel disease in group $A$ is $36.8 \%(n=40)$ patients which is significantly higher as compared to group B i.e11.4\% ( $\mathrm{n}=11)$ patients. (Table 4)

Table-4: Comparison of numbers of vessels affected in inferolateral ami \& inferior wall ami

\begin{tabular}{|c|c|c|c|c|c|}
\hline \multirow{2}{*}{ NUMBER OF CORONARY VESSEL EFFECTED } & \multicolumn{2}{|c|}{ IWMI+LMI } & \multicolumn{2}{|c|}{ IWMI ONLY } & \multirow{2}{*}{ P-VALUE } \\
\cline { 2 - 5 } & $(\mathbf{n o s})$ & $(\boldsymbol{\%})$ & $(\mathbf{n o s})$ & $(\boldsymbol{\%})$ & \\
\hline SVD & 69 & 63.2 & 85 & 88.6 & $<0.001$ \\
\hline DVD & 22 & 20.2 & 7 & 7.26 & 0.0076 \\
\hline TVD & 18 & 16.6 & 4 & 4.16 & $>0.001$ \\
\hline
\end{tabular}

7) When SVD taken into account, LCX is main culprit artery in $69 \%(n=48)$ patients in inferolateral MI (Group A), where as in inferior wall MI (Group B) RCA is the main culprit artery in $83 \%(n=71)$ patients. (Table 5)

Table-5: Culprit vessel in inferior and inferolateral mi -a comparison study

\begin{tabular}{|c|c|c|c|c|}
\hline CULPRIT & \multicolumn{2}{|c|}{ LCX } & \multicolumn{2}{c|}{ RCA } \\
\cline { 2 - 5 } VESSEL & (NOS) & $(\%)$ & $($ nos $)$ & $(\%)$ \\
\hline IWMI+LMI & 48 & 69.5 & 21 & 30.4 \\
\hline IWMI & 14 & 16.4 & 71 & 83.5 \\
\hline P-VALUE & \multicolumn{2}{|c|}{$<0.0001$} & \multicolumn{2}{c|}{$<0.0001$} \\
\hline
\end{tabular}

\section{DiscussiOn}

In our study, lateral wall MI present in $33.8 \%$ patients with inferior wall MI. ST-depression with Qwave equivalent seen in v1, v2 around $46 \%$ patients.
ST elevation in leads like I,avl and V5,V6 seen in around $35 \%$ patients. Lateral wall ECG changes in both group of leads i.e v1,v2,v5,v6,I,avL seen in $18.9 \%$ of patient with inferolateral MI. Peterson et al. found out that $38.9 \%$ patients with inferior wall MI have ST depression in V1 \&V2[3]. Whereas our studies found out to be $36.2 \%$. Adawi and Atar et al. studied in inferior wall MI that, $68(57 \%)$ patients had inferior wall ECG changes only, and $51(42.8 \%)$ patients had inferior with lateral wall involvement (i.e. leads I, AVL and/or V5-V6)[4]. However inclusion criteria for both the above study are different. Our study included STEMI equivalent in $\mathrm{V}_{1} \mathrm{~V}_{2}$ in addition lead $\mathrm{I}, \mathrm{AV}_{\mathrm{L}}$ and $\mathrm{V}_{5} . \mathrm{V}_{6}$, which is not included in the adawiatar et al. study.

Mean EF of inferolateral MI patients at the time of presentation also significantly lower as compared to inferior wall MI (group B). $51.9 \%$ of 
patient of inferolateral MI have normal systolic function, $29.8 \%$ patients have mild LV systolic dysfunction, $16 \%$ have moderate LV dysfunctionand $2.1 \%$ have severe LV dysfunction. On the other hand in inferior wall MI only $9 \%$ patients have LV dysfunction, out of which $8.1 \%$ patients have mild LV dysfunction \& $0.9 \%$ Patients has mod LV dysfunction. No patients in inferior wall MI (group B) have any form of severe LV dysfunction.So it is concluded that patients with inferior wall MI (group B) rarely have mod to severe LV dysfunction. Also it is seen that, our findings are consistent with findings of other studies. Oraiis, Maleki-etal have also found out that patient with tall $\mathrm{R}$ wave in V1, V2 with inferior wall MI have lower $\mathrm{EF}$, more complications and increased in hospital mortality [5]. Matetzky et al. found high peak creatinine kinase (with $\mathrm{p}$ value less than 0.02), lower LVEF at hospital discharge $(\mathrm{p}<0.008)$ [6] in patient with posterior wall motion abnormality during radionuclide angiography [6].

In our study, we have found more incidence of mitral regurgitation with statistical significance. Adawi and Atar et al. found that, inferolateral MI patient have higher incidence significant $\mathrm{MR}$, which coincide to finding in our study [4]. The cause of MR may be due to more incidence of posteromedial papillary muscle rupture or dysfunction as a result of single blood supply to it. As more area of myocardium are geopardised in inferolateral MI group leading to abnormal contraction pattern, LV dilation \& subsequently papillary muscle are displacement, which may be an explanation for MR.

Out of 109 patients of inferolateral MI, 96 patients underwent angiography. To avoid confusion while finding the culprit vessel, SVD lesion taken into account for the study. It is seen that, LCX is the culprit lesion in $69.5 \%$ patients with inferolateral MI. However RCA is the culprit lesion in $83.5 \%$ inferior wall MI patients. Kulkarni-et al. observed in his study that ST elevation in lateral and posterior leads was most frequently seen during LCX inflation but STelevation in inferior leads alone was not seen in LCX inflation [7].

Surprisingly it is seen that $36.8 \%$ of patient in group-A have more than 1 vessel disease as compared to $11.4 \%$ in group-B with statistical significance $(\mathrm{p}<0.001)$. These findings are more consistent with finding of Birnbaum-etal, who found that, ST-depression in anterior precordial leads in inferior wall MI are associated with more incidence of multivessel disease[8]. Also Peter B. Berger- etal found that 25 patients out of 53 patients with acute inferior infarction accompanied by ST depression in anterior leads have multivessel disease [9].

LV dysfunction, mitral regurgitation and multivessel involvement lead to complications like LVF, cardiogenic shock, arrythmia. Eric. D. Peterson found out in his study that patients with lateral wall changes in $\mathrm{V} 1, \mathrm{~V} 2$ have more post infraction complications like CHF or shock and higher mortality as compared to patient without ST-depression in V1,V2[3]. So patient with lateral wall ECG changes presents with more incidence cardiovascular complications and mortality as compared to inferior wall MI without lateral wall ECG changes.

\section{Conclusion}

Around $1 / 3$ rd patients with inferior wall STEMI have ECG changes of lateral wall AMI. STEMI equivalent in lead V1,V2 which was previously ignored in other studies, constitute about $2 / 3^{\text {rd }}$ of entity. Around half of the Inferolateral MI patients have LV systolic dysfunction. LCX is main culprit artery, but multivessel disease seen in more than $1 / 3^{\text {rd }}$ cases, which is comparatively higher indicating grave prognosis. As patient's presents with LV dysfunction, multivessel disease and mitral regurgitation, so it has grievous impact on clinical outcome. Meticulous attention should be given to the ECG changes in lateral leads in inferior wall MI to increase the efficacy of emergency management strategy. Limitation of Study: Small number of patient studied. Further subgroup analysis required in inferolateral $\mathrm{MI}$.

\section{REFERENCES}

1. Bayes de Luna A, Wagner G, Birnbaum Y, Nikus K, Fiol M, Gorgels A, Cinca J, Clemmensen PM, Pahlm O, Sclarovsky S, Stern S. A new terminology for left ventricular walls and location of myocardial infarcts that present $Q$ wave based on the standard of cardiac magnetic resonance imaging: a statement for healthcare professionals from a committee appointed by the International Society for Holter and Noninvasive Electrocardiography. Circulation. 2006 Oct 17;114(16):1755-60.

2. Bayés de Luna A. New heart wall terminology and new electrocardiographic classification of Q-wave myocardial infarction based on correlations with magnetic resonance imaging. Revista espanola de cardiologia. 2007 Jul 1;60(7):683-9.

3. Peterson ED, Hathaway WR, Zabel KM, Pieper KS, Granger CB, Wagner GS, Topol EJ, Bates ER, Simoons ML, Califf RM. Prognostic significance of precordial ST segment depression during inferior myocardial infarction in the thrombolytic era: results in 16,521 patients. Journal of the American College of Cardiology. 1996 Aug 1;28(2):305-12.

4. Adawi K, Atar S. Clinical implications and angiographic and electrocardiographic correlation of ST segment elevation in leads V7-V9 in patients with ST elevation myocardial infarction. Harefuah. 2008 Jul 1;147(7):587-90.

5. Oraii S, Maleki M, Tavakolian AA, Eftekharzadeh M, Kamangar F, Mirhaji P. Prevalence and outcome of ST-segment elevation in posterior 
electrocardiographic leads during acute myocardial infarction. Journal of electrocardiology. $1999 \mathrm{Jul}$ $1 ; 32(3): 275-8$.

6. S Matetzky1, D Freimark, $\mathrm{P}$ Chouraqui. Significance of ST Segment Elevations in Posterior Chest Leads $\left(\mathrm{V}_{7}\right.$ to $\left.\mathrm{V}_{9}\right)$ in Patients with Acute Inferior Myocardial Infarction: Application for Thrombolytic Therapy. JACC. 1998; 31(3), 506511

7. Kulkarni AU, Brown R, Ayoubi M, Banka VS. Clinical use of posterior electrocardiographic leads: a prospective electrocardiographic analysis during coronary occlusion. American heart journal. 1996 Apr 1;131(4):736-41.

8. Birnbaum Y, Drew BJ. The electrocardiogram in ST elevation acute myocardial infarction: correlation with coronary anatomy and prognosis. Postgraduate medical journal. 2003 Sep 1;79(935):490-504.

9. Peter B. Berger, and Thomas J. Ryan; Clinical Progress Series of Inferior Myocardial Infarction in High-Risk Subgroups: AHA.1990 Feb; 81(2):40111doi; http;//ahajournals.org on November 18, 2020 . 